\title{
Níveis de chumbo e cádmio em suplementos minerais para bovinos comercializados em Londrina*
}

\section{Levels of lead and cadmium in mineral supplements for cattle commercialized in Londrina}

\author{
Wilmar Sachetin Marçal ${ }^{1}$; Iara de Oliveira Buture²; Marcos Coelho de Carvalho³; \\ Maíra Salomão Fortes ${ }^{3}$; Rodrigo de Andrade e Silva ${ }^{4}$
}

\begin{abstract}
Resumo
Para se proceder a uma investigação em misturas minerais, foi realizada uma pesquisa quantificando os elementos chumbo e cádmio em diferentes formulações, comercializadas na cidade de Londrina. Os metais pesados foram determinados pela técnica de espectrofotometria de absorção atômica por plasma de indução acoplada. Considerando como parâmetro de comparação às referências do National Research Council (1996) e a Association of American Feed Control Officials Incorporated (2001), em 10 diferentes marcas analisadas, seis apresentaram níveis de chumbo inorgânico superiores ao limite máximo aceitável que é de $30 \mathrm{ppm}$. Contudo, em todas as amostras o nível de cádmio encontrado superou o limite de 0,5 ppm, que é o valor máximo aceitável. Os resultados demonstram a urgente necessidade de monitoramento junto aos fabricantes e/ou revendedores, pois algumas misturas minerais podem ser eminentemente perigosas, possibilitando efeitos cumulativos tóxicos de chumbo e cádmio inorgânico aos bovinos.

Palavras-chave: Metais pesados, chumbo, cádmio, suplementos minerais, bovinos.
\end{abstract}

\begin{abstract}
We measured lead and cadmium concentrations in samples of mineral mixtures commercialized in Londrina city. Lead and cadmium content were determined by inductively coupled plasma atomic emission spectrometry. Considering maximum concentrations recommended by National Research Council (1996) and Association of American Feed Control Officials Incorporated (2001), of the 10 analyzed samples, 06 had values greater than $30 \mathrm{ppm}$ of lead and all of them had values greater than $0.5 \mathrm{ppm}$ of cadmium. These findings show the necessity for careful industrial monitoring because some mineral mixtures contain sufficient lead and cadmium to cause bovine toxicity.
\end{abstract}

Key words: Heavy metals, lead, cadmium, mineral supplements, cattle.

\footnotetext{
* Trabalho realizado com apoio cultural da PROPPG-UEL .

1 Médico veterinário, Professor Associado do Departamento de Clínicas Veterinárias, Centro de Ciências Agrárias, Universidade Estadual de Londrina.E-mail: wilmar@uel.br

2 Médica veterinária, aluna do Curso de Pós-Graduação em Ciência Animal, UEL.

3 Acadêmico(a) de Medicina Veterinária, bolsista do Programa PIBIC/IC, UEL.

4 Acadêmico(a) de Medicina Veterinária, estagiário no Projeto Xenobióticos (PROPPG-UEL).
} 
A região norte do Paraná é detentora de expressivo rebanho de bovinos, com exploração pecuária bem desenvolvida e tecnificada, o que propicia um significativo campo de negócios para os que sobrevivem da cria, recria e engorda dos animais. Para fomentar toda essa atividade há um grande mercado consumidor no campo da suplementação mineral, sobretudo em Londrina, por se tratar de uma cidade pólo em contínuo crescimento.

Todavia, visando baratear custos para ganhar mercado e garantir suas vendas, algumas indústrias produtoras e/ou misturadoras de sal mineralizado para bovinos utilizam fontes de matérias-primas escolhidas pelo preço mais acessível. Por essa razão, acreditase que algumas formulações minerais possam estar contaminadas por elementos tóxicos, sobretudo metais pesados e substâncias radioativas. Essa suspeita, aliada ao fato de que o governo brasileiro no ano de 2000, através do Ministério da Agricultura, Pecuária e Abastecimento (Portaria SRD n. ${ }^{\circ} 20$ de 06/07/1997), liberou o uso de fontes alternativas de fósforo a partir de fosfatos de rochas, reiteram a necessidade de se monitorar as formulações minerais destinadas ao consumo animal revendidas em qualquer ponto do país, incluindo Londrina.

Do ponto de vista econômico, diferentes autores destacam que os metais pesados, como o chumbo e o cádmio, quando presentes em suplementos alimentares para animais, podem causar alterações orgânicas importantes, modificando a performance dos animais (LOBÃO, 1977; MALETTO, 1986; SILVA, 1993; ASSOCIATION OF AMERICAN FEED CONTROL OFFICIALS INCORPORATED, 2001), podendo acarretar significativas alterações no sistema reprodutivo dos bovinos (McDOWELL, 1985; MARACEK et al., 1998), inclusive abortamento (STUART; OEHME, 1982; MARÇAL et al., 2001; MARÇAL et al., 2003).

É necessário, todavia, destacar a preocupação dos pesquisadores em vários locais do mundo, os quais demonstram a possibilidade de uma formulação mineral, contaminada por metais pesados, atingirem a cadeia trófica alimentar, atingindo os bovinos e, por conseguinte o homem, através do consumo de produtos e/ou subprodutos de origem animal notadamente tóxicos, o que representa potencial risco à saúde pública (MALETTO, 1986; ANDRIGUETTO et al., 1990; ALLEN, 1992; JUNQUEIRA, 1993; SILVA, 1993; CAMPOS NETO; MARÇAL, 1996; MARÇAL; CAMPOS NETO; NASCIMENTO, 1998; MARÇAL et al., 2001).

Os elementos escolhidos para esse estudo investigativo foram o chumbo e o cádmio, considerados por muitos estudiosos como sendo elementos químicos inorgânicos de maior risco à saúde dos animais de criação, particularmente na espécie bovina (KANEKO, 1989; ANDRIGUETTO et al., 1990; ALLEN, 1992; BRITO, 1993; VILLEGAS-NAVARRO et al., 1993; NATIONAL RESEARCH COUNCIL, 1996; MARÇAL et al., 1999; MARÇAL et al., 2001; MARÇAL et al., 2003).

Do ponto de vista toxicológico o chumbo é causa comum de intoxicação em bovinos, sobretudo animais jovens, normalmente associada à ingestão de alimentos contaminados. Os efeitos tóxicos manifestam-se por encefalopatia, gastroenterite e degeneração dos nervos periféricos, podendo se acumular em tecidos dos animais e ser eliminado pelo leite, o que representa riscos à saúde pública (RADOSTITS et al., 2000). Segundo Marçal et al. (1999) suplementos minerais com restrito controle de qualidade por parte dos fabricantes, podem conter chumbo suficiente para causar alterações clínicas importantes em bovinos, principalmente alterações reprodutivas como degeneração testicular em touros e acúmulo em ovários, causando aciclia em vacas e interferências no ciclo reprodutivo.

O cádmio, também considerado elemento de alta toxicidade, causa severas alterações patológicas como disfunção renal, tumor e necrose testicular, arteriosclerose, lesões no sistema nervoso central e inibição de crescimento em seres humanos e animais (ANDRIGUETTO et al., 1990). O cádmio ainda se acumula no leite, ovos e carne, sendo que sua concentração nos tecidos é proporcional a sua ingestão (SHIRLEY, 1985). Church e Pond (1977) 
ainda destacaram que o cádmio é perigoso para a saúde pública por causar problemas de esterilidade, lesões renais e testiculares, além de anemia. Além disso, Takebayashi et al. (2000) confirmaram que o cádmio foi responsável por problemas renais em pacientes humanos, induzindo osteomalácia pela atrofia no túbulo proximal com alterações na reabsorção de fósforo.

Neste aspecto, o objetivo do presente trabalho foi investigar a presença de chumbo e cádmio em diferentes suplementos minerais para bovinos, mais comercializados na cidade de Londrina, buscando através de análises laboratoriais, quantificar contaminantes que possam estar agregados aos elementos minerais, nas formulações preparadas para consumo animal.

\section{Colheita e análise das amostras de sal mineral}

As amostras de sal mineral foram colhidas diretamente do estoque disponível em estabelecimentos comerciais, além de propriedades rurais colaboradoras. As amostras foram acondicionadas em recipientes de plástico transparente, previamente identificados, com aproximadamente 200 gramas de cada diferente marca. O método de amostragem utilizado seguiu as recomendações da Andif (1997). As análises foram efetuadas no Laboratório Rodes Química, na cidade de Cajati, estado de São Paulo.

$\mathrm{Na}$ metodologia analítica empregada para a determinação dos elementos no sal mineral, as amostras foram previamente secas a $110^{\circ} \mathrm{C}$ por aproximadamente duas horas. A solubilização foi feita com os ácidos nítrico, perclórico e fluorídrico. Procedeu-se a determinação dos metais por espectrofotometria de absorção atômica por plasma de indução acoplada, empregando-se um equipamento Varian, modelo 220 FS, de alta sensibilidade. Os elementos chumbo e cádmio foram separados da amostra por extração com pirrolidina ditiocarbamato de amônia (APDC) p.a. em pH 2,3 $\pm 0,1$. Esta metodologia de análise empregada, baseia-se no manual da American Society for Testing and Materials (1980) e na descrição de Eaton, Greenberg e Trussell (1995). As análises foram feitas por uma única amostragem, pois o laboratório mantém padrão de excelência no controle de qualidade dos equipamentos, com aferições e calibragens constantes, minimizando custos e evitando a necessidade de repetibilidade de mensurações, devido inclusive ao alto custo operacional das análises. $\mathrm{O}$ limite mínimo de determinação do método para o chumbo é 1,5 ppm e para o cádmio é 0,5 ppm.

Os resultados obtidos na presente pesquisa, na qual se quantificou os elementos inorgânicos chumbo e cádmio nas formulações minerais mais comercializadas na cidade de Londrina, são apresentados na Tabela 1. Pode-se perceber que, os valores encontrados de chumbo variaram de $16 \mathrm{ppm}$ (amostra L 06) a 363 ppm (amostra L 09) e somente quatro amostras analisadas apresentaram resultados de chumbo inorgânico inferiores aos 30 ppm, representando $40 \%$ das formulações investigadas. Todavia, com relação ao cádmio, todas as amostras analisadas apresentaram níveis elevados desse metal pesado, acima do limite máximo aceitável $(0,5 \mathrm{ppm})$ pela normatização vigente.

Tabela 1. Resultados da análise laboratorial para quantificação dos elementos inorgânicos chumbo e cádmio em diferentes marcas de sal mineral, comercializadas na cidade de Londrina, estado do Paraná, 2004.

\begin{tabular}{ccc}
\hline n. $^{\mathbf{0}}$ da amostra & $\begin{array}{c}\text { valores de } \\
\text { chumbo } \\
\text { em ppm }\end{array}$ & $\begin{array}{c}\text { valores de } \\
\text { cádmio } \\
\text { em ppm }\end{array}$ \\
\hline L 01 & 47 & 6 \\
L 02 & 28 & 4 \\
L 03 & 39 & 5 \\
L 04 & 28 & 10 \\
L 05 & 27 & 57 \\
L 06 & 16 & 3 \\
L 07 & 35 & 4 \\
L 08 & 58 & 9 \\
L 09 & 363 & 6 \\
L 10 & 38 & 2 \\
\hline
\end{tabular}


A proposta deste estudo foi investigar a presença dos elementos chumbo e cádmio nos suplementos minerais, misturados e/ou comercializados em Londrina, visando, dar contribuição às ações de rastreabilidade e produção orgânica de bovinos, principalmente quando o marketing das exportações brasileiras propaga o "boi verde". A té então não havia trabalho desta natureza, considerando as dez formulações mais comercializadas na cidade. Por isso, ressaltou-se a necessidade desta investigação, já que o Ministério da Agricultura, Pecuária e Abastecimento ao que se sabe, oficiosamente, não detém instrumento prático de fluxo contínuo que atenda esse objetivo no município (PANSARD, 2002).

Nesta investigação sobre o chumbo e cádmio utilizaram-se as formulações minerais já misturadas, porque não foi possível separar as matérias-primas para investigar cada um de seus componentes. Portanto, trabalhou-se com as formulações industrializadas prontas. A suspeita maior da presença de metais pesados nas misturas minerais é a de que estejam incorporados às fontes de macroelementos, sobretudo fósforo, porque este mineral representa o maior custo na composição de um sal mineral (SOUSA, 1981; ROSA, 1989). Por isso, os fabricantes buscam esse elemento essencial em fontes alternativas mais baratas, como por exemplo, nos fosfatos naturais de rocha (AMMERMAN; MILLERE; FICK, 1977; VIANA, 1985; ROSA, 1989; CAMPOS NETO, 1992; MARÇAL et al., 1999), ou no ácido fosfórico importado (BRITO, 1993; MARÇAL; CAMPOS NETO; NASCIMENTO, 1998). Isto pode tendenciar outras pesquisas com investigação dirigida. Todavia, deve ser lembrado que esses metais pesados podem também ser oriundos de matérias-primas de microelementos (CAMPOS NETO; MARÇAL, 1996).

Os resultados destacados na Tabela 1 demonstram que em seis das 10 diferentes amostras, os valores de chumbo inorgânico extrapolaram o limite máximo aceitável de $30 \mathrm{ppm}$ referenciado pelo National Research Council (1996) e Association American of Feed Control Incorporated (2001).
Porém, e tendo as mesmas referências como base de comparação, em todas as amostras analisadas os valores de cádmio extrapolaram o limite máximo aceitável de 0,5 ppm.

Com a quantificação dos dois elementos tóxicos da presente pesquisa, a próxima etapa poderá oportunizar a investigação da performance dos rebanhos consumidores dos sais minerais contaminados. É sabido que há aspectos subclínicos ou silenciosos que devem ser observados como alerta: os bovinos que consumem sal mineral com níveis considerados tóxicos pela presença de chumbo e cádmio podem ter alterações no sistema reprodutivo, interferências no ciclo reprodutivo das vacas, anestro, aumento no intervalo entre-partos e alterações de performances, como referem Stuart e Oehme (1982), McDowell (1985), Maracek et al. (1998), Marçal et al. (1999) e Marçal et al. (2001).

Outro ponto a ser explorado pela pesquisa científica diz respeito ao antagonismo mineral, pois o chumbo e cádmio quando presentes em suplementos minerais induzem a deficiência de elementos essenciais da dieta mineral dos bovinos, principalmente o cálcio (BARTON; CONRAD; HARRISON, 1978; MARÇAL, 1996; NATIONAL RESEARCH COUNCIL, 1996; MARÇAL et al., 2003). Ainda é importante salientar que o chumbo e o cádmio antagonizam as ações do zinco, que é um mineral essencial no metabolismo de algumas enzimas (MARÇAL et al., 2003).

Ainda é preciso destacar que há uma preocupação ambientalista da presença de metais pesados em suplementos minerais de bovinos. A ingestão de elementos inorgânicos em grandes quantidades, causada pelo alto índice de contaminação dos suplementos, leva os bovinos a defecarem expressiva quantidade de fezes diariamente. Isto pode significar, em curto prazo, a contaminação itinerante do solo, da vegetação, das fontes de água de beber e de diferentes formas de seres vivos do ecossistema.

Pelos resultados encontrados, pode-se concluir que há sólidos indícios técnico-científicos de que está 
ocorrendo a comercialização, na cidade de Londrina, de formulações minerais para bovinos contaminadas por chumbo e cádmio inorgânicos. Ainda é possível concluir que se os órgãos fiscalizadores e as indústrias misturadoras de sal mineral não se tornarem mais rigorosas no controle de pureza das matérias-primas na composição das formulações e, por outro lado, havendo o crescimento da comercialização desses insumos, haverá possibilidade da presença de mais contaminantes na alimentação animal, no meio ambiente e infelizmente, atingindo o homem, através da cadeia alimentar comprometida. Enfim, esses fatos certamente poderão originar pontos fortemente negativos no competitivo mercado comercial, sobretudo nas exportações de carne brasileira.

\section{Referências}

ALLEN, J. D. Minerals in animal feed. Industrial Minerals, London, n.292, p.35-39, 1992.

AMERICAN SOCIETY FOR TESTING AND MATERIALS. Annual book of ASTM Standards. Philadelphia, 1980

AMMERMAN, C. B.; MILLERE, S. M.; FICK, K. R. Contaminating elements in mineral supplements and their potential toxicity: a review. Journal of Animal Science, Champaign, v.44, n.3, p.485-508, 1977.

ANDIF. O fósforo na alimentação animal. Associação Nacional para Difusão de Fontes de Fósforo na Alimentação Animal. São Paulo, 1997, 74p.

ANDRIGUETTO, J. M.; PERLY, L.; MINARDI, I.; GEMAEL, A.; FLEMING, J. S.; SOUZA, G. A.; BONA FILHO, A. Os princípios nutritivos e suas finalidades. In: . Nutrição animal. 4. ed. São Paulo: Nobel, 1990. $\overline{\text { p.189-255. }}$

ASSOCIATION OF AMERICAN FEED CONTROL OFFICIALS INCORPORATED. Official guidelines for contaminant levels permitted in mineral feed ingredients. Indiana, 2001

BARTON, J. C.; CONRAD, M. E; HARRISON, L. Effects of calcium on the absorption and retention of lead. Journal of Laboratory Clinical Medicine, Saint Louis, v.91, p.366$76,1978$.

BRITO, J. Fosfato bicálcico feed grade. Cajati: Serrana, 1993. Apostila mimeo.
CAMPOS NETO, O. Pesquisa esclarece dúvidas sobre déficit na nutrição animal. O Corte, São Paulo, v.24, p.14, 1992.

CAMPOS NETO, O.; MARÇAL W. S. Os fosfatos na nutrição mineral de ruminantes. Revista dos Criadores, São Paulo, n.793, p.8-10, 1996.

CHURCH, D. C.; POND, W. G. Bases científicas para la nutricion y alimentación de los animales domésticos. Zaragoza: Acríbia, 1977.

EATON, C. L. S.; GREENBERG, A. E.; TRUSSELL, R. R. (Ed.). Standard methods for the examination of water and wastewater. Washington: APHA, 1995.

JUNQUEIRA, O. M. Metais pesados contaminam carne. Avicultura \& Suinocultura Industrial, São Paulo, n.38, p.27-29, 1993.

KANEKO, J. Clinical biochemistry of domestic animals. 4. ed. New York: Academic Press, 1989.

LOBÃO, A. O. Mineralização de bovinos de corte. In: SIMPÓSIO SOBRE PECUÁRIA DE CORTE, 1977, Presidente Prudente. Anais... Jaboticabal: UNESP, 1977. p.120-135.

MALETTO, S. Correlação da nutrição mineral e a sanidade. In: SEMINÁRIO SOBRE NUTRIÇÃO MINERAL, 1986, São Paulo. Anais... São Paulo: Instituto Brasileiro do Fósforo, 1986. p.38.

MARACEK, I.; LAZAR, L.; DIETZOVA, I.; KORENEKOVA, B.; CHOMA, J.; DAVID, V. Residues of heavy metals in cow reproductive organs and morbidity of cattle in the fallout region of a metallurgical plant. Veterinary Medicine, Praga, v.43, n.9, p.283-287, 1998.

MARÇAL, W. S.; GASTE, L.; NASCIMENTO, M. R. L.; LIBONI, M.;GOMES, G. P.; HISASI, C. S. Cadmium concentration in mineral salt mixtures used as supplementation in beef cattle food. Veterinarski Arhiv, Zagreb, v.73, n.1, p.47-53, 2003.

MARÇAL, W. S. Valores sangüineos de bovinos nelore em pastejo de Brachiaria decumbens, suplementados com sal mineral naturalmente contaminado por chumbo. 1996. Tese (Doutorado) - Faculdade de Medicina Veterinária e Zootecnia, Universidade Estadual Paulista, Botucatu.

MARÇAL, W. S.; GASTE, L.; LIBONI, M.; PARDO, P. E.; NASCIMENTO, M. R. L. Concentration of lead in mineral salt mixtures used as supplements in cattle food. Experimenthal and Toxicologic Pathology, Jena, v.53, p.7-9, 2001. 
MARÇAL, W. S.; GASTE, L.; LIBONI, M.; PARDO, P. E.; NASCIMENTO, M. R. L. Lead Concentration in mineral salt mixtures used in beef cattle food supplementation in Brazil. Veterinarski Arhiv, Croatia, v.69, n.6, p.349-355, 1999.

MARÇAL, W. S.; CAMPOS NETO, O.; NASCIMENTO, M. R. L. Valores sangüíneos de chumbo em bovinos Nelore suplementados com sal mineral naturalmente contaminado por chumbo. Ciência Rural, Santa Maria, v.28, n.1, p.5357, 1998 .

McDOWELL, L. R. Nutrition of grazing ruminants in warm climates. Orlando: Academic Press, 1985.

NATIONAL RESEARCH COUNCIL. Subcommittee on Mineral Toxicity in Animals. Nutrient requirements of beef cattle. Washington: National Academy of Science, 1996. p.256-76.

PANSARD, N. Informações sobre fiscalização em suplementos minerais pelo Ministério da Agricultura. Londrina, 2002. (Comunicação Pessoal).

RADOSTITS, O. M.; GAY, C. C.; BLOOD, D. C.; HINCHCLIFF, K. W. Clínica veterinária: um tratado de doenças dos bovinos, ovinos, suínos, caprinos e eqüinos. 9. ed. Rio de Janeiro: Guanabara Koogan, 2000. p.14191423.

ROSA, I. V. Fosfato natural como suplemento de fósforo para bovinos. In: VALLE, E. R. et al. Coletânea de seminários técnicos 1986/88. Campo Grande: Embrapa, 1989. p.59.
SHIRLEY, R. L. Water requirements for grazing ruminants and water as a source of minerals. In: McDOWELL, L. R. Nutrition of grazing ruminants in warm climates. Orlando: Academic Press, 1985. p.182-186.

SILVA, S. Plano de ação fiscal sobre fosfato de rocha e outros. Brasília: Ministério da Agricultura, do Abastecimento e da Reforma Agrária, 1993.

SOUSA, J. C. Aspectos da suplementação mineral de bovinos de corte. Campo Grande: EMBRAPA/CNPG, 1981. (Circular Técnica, 5).

STUART, L. D.; OEHME, F. V. Environmental factors bovine and porcine abortion. Veterinary and Human Toxicology, Manhattan, v.24, p.435-41, 1982.

TAKEBAYASHI, S.; JIMI, S.; SEGAWA, M.; KIYOSHI, Y. Cadmium induces osteomalacia mediated by proximal tubular atrophy and disturbances of phosphate reabsorption: a study of 11 autopsies. Pathology Research and Practice, Stuttgart, v.196, n.9, p.653-663, 2000.

VIANA, J. A. C. Fontes de sais minerais para bovinos e o desafio de suplementos de fósforo no Brasil. In: SIMPÓSIO SOBRE NUTRIÇÃO DE BOVINOS, 3., 1985, Piracicaba. Anais... Piracicaba: FEALQ, 1985.

VILLEGAS-NAVARRO, A.; BUSTOS, O. D. M. E.; REYES, A. R.; DIECK, T. A.; REYES, J. L. Determination of lead in paired samples of blood and synovial fluid of bovines. Experimental and Toxicologic Pathology, Jena, v.45, p.479, 1993. 